\title{
COVID -19: EFFICACY AND SAFETY PROFILE OF MAIN VACCINES APPROVED FOR EMERGENCY USE AUTHORIZATION IN 2021
}

\author{
Haghamad Allzain Mohamed Bulla ${ }^{1}$ 四 (1) \\ ${ }^{1}$ Assistant Professor In The Department Of Biochemistry, Faculty Of Medicine, Shendi University, Sudan
}
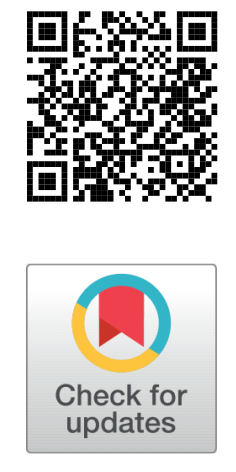

Received 1 July 2021

Accepted 12 July 2021

Published 31 July 2021

Corresponding Author

Haghamad Allzain Mohamed

Bulla,Hajamadbulla@Yahoo.Com

DOI 10.29121/

granthaalayah.v9.i7.2021.4062

Funding: This research received no specific grant from any funding agency in the public, commercial, or not-for-profit sectors.

Copyright: (C) 2021 The Author(s). This is an open access article distributed under the terms of the Creative Commons Attribution License, which permits unrestricted use, distribution, and reproduction in any medium, provided the original author and source are credited.

\section{ABSTRACT}

Coronavirus -2 (SARS-CoV-2), is a new member of the human coronaviruses family. It is a single-strand positive-strand nucleic acid (ssRNA), It also has spike (S), membrane (M), envelope (E), and nucleocapsid (N) proteins. The $(\mathrm{S})$ protein is responsible for recognising and binding to receptors on the surface of host cells and plays an important role in the first step of viral infection. Coronavirus- 2 is identified in late 2019 as the causative agent of a new disease (COVID-19) outbreak in China associated with severe medical complications and even death in some cases. In March 2020, the World Health Organization (WHO) announced the novel disease outbreak as a pandemic. Current Coronavirus pandemic is the most challenging health emergency to humanity in this century, with globally reported (184 .324 .026) confirmed cases and (3. 992. 680 ) confirmed deaths ,as of $7^{\text {th }}$ July 2021, according to WHO.

Preventive measures of social distancing face masks wearing, hand washing and lockdown have slowed the spread of COVID-19, but the ideal one is vaccination to protect individuals and create everlasting change and return to normalcy. Efficacy and safety of vaccines is very essential to gain public trust.

There is a great variation in people's confidence in vaccines that relies on several factors, including awareness about vaccines, its side effects, safety and efficacy.

The objective of this review is to provide concise sound knowledge concerning the main approved vaccines for protection against COVID -19 emerged from various phases of vaccines clinical trials and medical practices, to enhance public trust and to tackle vaccine skepticism .

This study also intended to educate about the vaccine's side effects, that may prompt individuals to receive the vaccine after comparing the acceptable side effects with the disease's severity and there by promote communities confidence in the safety of COVID-19 vaccines.

This review examines efficacy and safety profile of some authorized vaccines such as Pfizer/BioNTech mRNA, (BNT162b2), Moderna (mRNA-1273), Johnson \& Johnson (Janssen), Oxford-AstraZeneca ChAdOx1-S, CoronaVac vaccine and Gam-COVID-Vac (Sputnik V). 
The review concluded that currently authorized COVID-19 vaccines are effective and prevent serious COVID-19 symptoms and related hospitalization and death. They are safe with acceptable minor local and systemic side effects, reported by some recipients' of the vaccine and are similar to the known side effects encountered with previously known vaccines such as seasonal influenza vaccine, as expected outcomes of immune response to the vaccines. The reported serious side effects were very rare.

\section{Abbreviations}

COVID-19; coronavirus disease-2019. WHO ; World Health Organization. DNA ; Dexoyribonucleic Acid, RNA ; Ribonucleic Acid ; CDC; Centers for Disease Control and Prevention .FAD; Food and Drugs Administration, EMA ;European Medicine Agency . EUA; Emergency Use Authorization . EUL ; Emergency Use Listing. UK; United Kingdom . US ; United States. ROCCA; RSM Observatory for COVID vaccination Campaign monitoring Adverse events. COVAX; COVID19 Vaccines Global Access.

Keywords: Covid-19, Vaccine, Efficacy, Safety, Pfizer-Biontech, Astrazenca, Moderna, Johnson \& Johnson, CoronaVac, Sputnik V

\section{INTRODUCTION}

COVID-19 impacted vulnerable populations with worsen socioeconomic burden. Vaccines offer potential way to bring this pandemic to an end, therefore vaccine development and clinical trial emerged as a key research priority. The clinical trial will enable informed decision making when advocating for adoption of specific vaccine. It needs critical competency for its conduction and following strict WHO guidelines, which include ethical guidelines requiring informed consent for anyone participating in the trials.

There are over two hundred (200) COVID-19 vaccines under various stages of development and testing. A small number have progressed into clinical phases and getting national and international regulatory bodies' authorization to be administered.

Vaccine against COVID-19 is a remarkable scientific achievement of the century , developed and tested over very short time within one year, with optimistic hope to end this pandemic soon with starting of vaccines roll-out. Since there is limited information regarding risks of the new vaccines, spread of misinformation undermined public confidence in established authorities; hence participants may suffer before vaccination from anxiety and hesitancy. Public concerns about the longerterm safety of the new vaccines should be addressed. Since the mRNA technique is new in vaccine manufacturing, no one predicts its consequences. Confidence in any COVID-19 vaccine will be crucial to its success. The appropriate pausing of any clinical trial, to carefully investigate safety concerns generated much publicity despite the reassuring outcomes of the safety review and trial recommencement Knoll and Wonodi (2021). 
Continuous monitoring for rare and common adverse effects experienced after vaccination is important for assessment of risks and benefits for each authorized COVID-19 vaccine. The innovative biotechnology industry culminated in the design and development of various types of COVID-19 vaccines, such as peptide, virus-like particle, replicating and non-replicating viral vectors, DNA or RNA, live attenuated virus, recombinant designed proteins and inactivated virus Seyed et al. (n.d.).

The vaccines may have other benefit, by reduction the transmitting of the virus. It is reported that one dose of vaccine reduces the potential for transmission by at least( $61 \%$ ), possibly considerably more, highlighting vaccine's efficacy in reducing viral positivity. Also the claim of high efficacy of mixed-dose regimen, need further study, as well as the efficacy of vaccine in older people.

Lipsitch and Kahn (2021).The COVID-19 vaccine doses administered globally were (3 .032. 056. 355) doses as of $7^{\text {th }}$ July 2021, according to WHO.

\section{Pfizer/BioNTech mRNA vaccine (BNT162b2)}

This new vaccine is based on messenger RNA (mRNA), which is genetic material that code for viral spike proteins found on SARS-CoV-2 virus, the causative agent for COVID-19 pandemic. Spike (S), proteins are used by the virus to gain entry into the human .The vaccine must be store at ultra-low temperatures of below ($70^{\circ} \mathrm{C}$ ) Mahase (2021d), making distribution challenge for many countries, because that temperature is much lower than that for most existing vaccines, kept between $\left(2-8^{\circ} \mathrm{C}\right)$.

Pfizer/ BioNTech mRNA vaccine (BNT162b2) is more than 90\% efficacious at preventing disease and is well-tolerated.

The UK became the first country to implement a COVID-19 vaccination programme after the approval of the Pfizer-BioNTech mRNA vaccine, BNT162b2, for emergency use. Bernal et al. (2021).

This vaccine was also approved by the U.S. FDA on the 11th of December2020 for EUA in individuals older than 16 years of age.

A single dose of the BNT162b2 vaccine is about (60-70\% ) effective at preventing symptomatic disease in adults aged( 70 ) years and older in England and that two doses are about (85-90\% ) effective. Those who were vaccinated and went on to have symptoms of COVID-19 had a (44\%) lower risk of being admitted hospital and a (51\%) lower risk of death compared with people who were unvaccinated. Bernal et al. (2021).

Other study found that a single dose of the Pfizer vaccine was associated with (58\%) protection against infection, (76\%) against hospital admission, and (77\%) against death. Iacobucci (2021), Ej et al. (2021), Leshem and Wilder-Smith (2021)

A consistently high efficacy of over .( 90 to $94 \%$ ) was observed in the clinical trials across age, sex, race, ethnicity and people with underlying medical conditions. after the second dose .and (82\%) after the first dose. Badiani et al. (2020). 
The first U.S. multisite test-negative design vaccine effectiveness study among health care personnel found a single dose of Pfizer-BioNTech or Moderna COVID19 vaccines to be (82\%) effective against symptomatic COVID-19 and two doses to be (94\%) effective. This clearly examined vaccine effectiveness in a real-world setting. Pilishvili et al. (2021)

Study conducted by Burd et al. (2021), provides evidence to support the safety and efficacy of COVID-19 vaccination in pregnancy with protection to the neonate against infection, outlining clear vaccine benefits for both maternal and child health.

Pfizer/ BioNTech mRNA vaccine (BNT162b2) is given in two shots, (21) days apart and recommended for anyone (12) years or older.

Most of the post-vaccination side effects were common and non-life-threatening

They are mild to moderate, sign that body's immune system is building protection, and similar to symptoms of most previous known vaccines Ma'mon et al. (n.d.). The common side effects are fatigue (90\%) and pain at the site of the injection (85\%)

Post Oxford-AstraZeneca and Pfizer-BioNTech vaccines side effects reported are not different from those that were reported in the clinical trials, indicating safe profile for both vaccines. They are highly consistent with the reported results in phase III clinical trials and fact sheets of the vaccines. Alhazmi et al. (2021). Other side effects include chills, headache, pain, tiredness, redness and swelling at the injection site, all of which subsided within a day or two of rest, with hydration, and medications like acetaminophen.

On rare occasions, mRNA vaccines have appeared to trigger anaphylaxis, For that reason, the $\mathrm{CDC}$ requires vaccination centers to monitor everyone for fifteen (15 Jminutes after their COVID-19 shot, and for thirty (30) minutes if they have a history of severe allergies or are taking anti-coagulants. Nagla et al. (2021).

Spectrum of skin reactions were reported after mRNA COVID-19 vaccines, with no reported serious adverse events. It should not discourage vaccination and it's not a contraindication to receiving the second dose of the Pfizer or Moderna vaccine. Health care workers must be aware of these potential vaccine reactions and advise patients accordingly. Counseling patients about potential benefits of receiving a COVID-19 vaccine is equally important. Mcmahon et al. (2021).

\section{Moderna mRNA-1273 vaccine}

It is mRNA- based vaccine, developed by US biotech company Moderna in partnership with the US National Institutes of Health (NIH). It can be stored in a household fridge for thirty (30) days, at room temperature for up to twelve (12) hours, and at $\left(-20^{\circ} \mathrm{C}\right)$ for up to six months. It is a clear advantage making it easier to distribute and store. Mahase (n.d.-c).

Moderna mRNA-1273 vaccine is a lipid nano-particle-encapsulated mRNA-based vaccine that encodes the perfusion stabilized full-length spike $(S)$ protein of the severe acute respiratory syndrome SARS-CoV-2 virus (coronavirus -2), that causes COVID-19. 
To promote public trust, vaccine designers- Jennifer Haller- receives the first dose of an mRNA vaccine to be administered, Cohen (2020). Moderna and Pfizer COVID19 vaccines are similar enough that can be swapped Vogel (2021).

On December 18, 2020, the Moderna mRNA-1273 vaccine was authorized by FDA for emergency use during the present COVID-19 pandemic in US. The UK has also approved the Moderna COVID-19 vaccine as a two dose regime, with the second dose given (28) days after the first one Mahase (n.d.-c).The time lapse between the two doses should not be more than six weeks, The second dose, can be given latter with no need to repeat the first dose, In special personal circumstances, the second dose can be given up to four days ahead of that schedule Edward and Livingston (2021)

The mRNA-1273 vaccine showed (94.1\%) efficacy after the second dose at preventing symptomatic COVID-19 illness, including severe disease. No safety concerns were identified, other transient local and systemic reactions, as suggested by the findings of phase (III) randomized, observer-blinded, placebo-controlled trial conducted at (99) centers across the U S, which enrolled (30.420) volunteers. The vaccine appeared to be equally effective across different ethnic and racial groups Baden et al. (2021). It is recommended to be used for persons aged eighteen (18) years and older. Oliver et al. (2021). Some experts believe it may provide protection against the Alpha, Beta and Delta, variants as the case with Pfizer vaccine since both are mRNA vaccines.

The initial post-authorization safety profiles did not indicate evidence of unexpected serious adverse events, as (90.9\%) of it were non-serious and involved local and systemic symptoms.These data provide reassurance and helpful information regarding what health care providers and vaccine recipients might expect after vaccination Gee et al. (2020).

The side effects of vaccination appear to be in line with those typically seen for other adult vaccinations, including the seasonal influenza vaccination. They were more commonly reported after the second dose than after the first one and were more frequent and severe in population aged (18-64) years than in those aged (65) years or more. They occurred within the first one to two days after vaccine receipt and subsided in a median of two to three days Oliver et al. (2021).

These side effects include chills, headache, pain, tiredness, and/or redness and swelling at the injection site and anaphylaxis on rare occasions (2.5 cases per million doses administered). In nine cases, anaphylaxis onset occurred within fifteen (15) minutes of vaccination. No anaphylaxis-related deaths were reported "Allergic Reactions Including Anaphylaxis After Receipt Of The First Dose Of Moderna Covid-19 Vaccine - United States" (2020).

For that reason, the $\mathrm{CDC}$ requires vaccination centers to monitor everyone for fifteen (15) minutes after their COVID-19 shot, and for thirty(30) minutes if they have a history of severe allergies. It is likely that vaccines that prevent symptomatic disease will reduce the duration and level of infectiousness, and thus reduce transmission, but it is not yet known if this effect will be large enough to make any significant dif- 
ference regarding the spread of the virus within communities Mahase (n.d.-a). Transient, pruritic rash that can present either as an urticarial welt or a macular erythematous patch in close proximity to the injection site seven to ten (7-10) days after the first dose was reported in a few cases

Other rare adverse effect is the "COVID arm", a localized, transient erythematous rash developed several days following the first dose of the Moderna COVID-19 vaccine. It is a benign condition, that should not deter from obtaining a second dose of the Moderna vaccine. Wei et al. (2021). Purpuric rash and thrombocytopenia after receiving the first dose of the m-RNA-1273 vaccine was also reported Malayala et al. (2021).

The CDC in US recommended that people who have had an allergic reaction to an ingredient in the vaccines should not receive the vaccine, while people who have not had allergic reactions to injectable vaccines but have had allergic reactions to food, pets, or medicines, but no anaphylactic reactions may receive it Tanne (2020).

\section{Johnson \& Johnson vaccine (Janssen vaccine)}

Janssen vaccine (Ad26.COV2.S), is a recombinant, non replicating adenovirus serotype 26 (Ad26) vector encoding a full-length and stabilized SARS-CoV-2 spike protein. Ad26-based vaccines are generally safe and highly immunogenic Sadoff, Gars, et al. (n.d.), Sadoff, Gray, et al. (n.d.).

Johnson \& Johnson has developed Janssen vaccine that works differently than the Pfizer and Moderna vaccines and is highly effective for preventing moderate to severe COVID-19. The company employed the same approach to make an Ebola vaccine that has been authorized for use by the EMA.

A single dose of Ad26.COV2.S protected against symptomatic COVID-19 and asymptomatic SARS-CoV-2 infection and was effective against severe critical disease, including hospitalization and death. Safety appeared to be similar to that in other phase (III) trials of Covid-19 vaccines. Sadoff, Gray, et al. (n.d.).

Since Janssen vaccine is based on a human adenoviral vector adenovirus type 26, a common cause of respiratory infections and It is based on stable DNA molecules, it does not require ultra cold storage, making it easier to distribute (Edward et al. (2021)).

The vaccine is estimated to remain stable for two years at $-20^{\circ} \mathrm{C}\left(-4^{\circ} \mathrm{F}\right)$, and a maximum of three months at routine refrigeration at temperatures of 2 to $8^{\circ} \mathrm{C}\left(36-46^{\circ} \mathrm{F}\right)$. The COVID-19 vaccine should not be re-frozen if distributed at temperatures of 2 to $8^{\circ} \mathrm{C}\left(36-46^{\circ} \mathrm{F}\right)$. Unpunctured vials may be stored between 9 to $25^{\circ} \mathrm{C}$ ( 48 to $77^{\circ} \mathrm{F}$ ) for up to twelve hours, and the vaccine can remain viable for months in a standard refrigerator. It is not shipped or stored frozen.

On February 27, 2021, the FDA issued an EUA for Janssen (Ad.26.COV2.S) COVID19 vaccine (Janssen Biotech, Inc., a Janssen Pharmaceutical company, Johnson \& Johnson) Shay et al. (2021). 
On March 12, 2021, the WHO issued an EUL for the Janssen (Ad26.COV2.S) COVID -19 vaccine .

The EMA of the European Commission approved the COVID-19 Vaccine Janssen on March 11, 2021.

Since Janssen is a single dose vaccine and has a lower cost, it is expected to play an important role in low and middle-income countries. It has been a popular vaccine to distribute to the homeless, prisoners, and refugee populations.

It is recommended for adults aged eighteen (18) years and older. The common side effects are Fatigue, fever, headache, pain at injection site, and myalgia They subside within one to two days. Sadoff, Gray, et al. (n.d.)

after administration of nearly eight (8) million doses of the Janssen COVID-19 vaccine. By April 21, 2021, review of safety monitoring data found that ( $97 \%$ ) of reported side effects after vaccine receipt were non-serious, and consistent with pre-authorization clinical trials data. Seventeen (17) thrombotic events with thrombocytopenia have been reported. Roll out of the vaccine was halted .On April 12, 2021, after detection of six cases of cerebral venous sinus thrombosis with thrombocytopenia, and resumed on April 23, 2021 after Advisory Committee on Immunization Practices in US concluded that the benefits of resuming Janssen COVID-19 vaccination among persons aged eighteen (18) years or older, outweighed the risks and reaffirmed its interim recommendation under FDA's EUA, with new warning for rare clotting events among women aged (18-49) years.. Macneil et al. (n.d.), Mahase (2021b)

\section{Oxford-AstraZeneca ChAd0x1-S vaccine}

It is based on chimpanzee adenovirus vectored DNA vaccine ChAdOx1 nCoV-19 (AZD1222) Knoll and Wonodi (2021).

A single dose of the ChAdOx1-S vaccine was about (60-75\%) effective against symptomatic disease and provided an additional protective effect against hospital admission. Bernal et al. (2021).

Interim analysis of data from four ongoing blinded, randomised, controlled trials done across the UK, Brazil, and South Africa, suggested acceptable safety profile and has been found to be efficacious against symptomatic COVID-19, with overall vaccine efficacy across (70.4\%), twenty one (21) days after the first dose, and in participants who received a low dose followed by a standard dose, efficacy was ( $90.0 \%$ ) Merrynvoyseydphil et al. (2021).

The vaccine can be stored at fridge temperature $\left(2-8^{\circ} \mathrm{C}\right)$ Mahase (n.d.-c).It is cost -effective and can be stored, transported, and handled in normal refrigeration for at least six months. It is administered as two doses, four to twelve (12) weeks apart and recommended for adults aged eighteen (18) years and older .

The common side effects reported are tenderness, pain, warmth, redness, itching, swelling or bruising at the injection site, all of which subsided within one to two days. Very rare blood clotting disorders were reported In April, 2021 EMA safety commit- 
tee concluded "unusual blood clots with low blood platelets should be listed as very rare side effects" that could occur within two weeks of receiving the vaccine.

The EMA has advised that information sheets on the vaccine for patients and healthcare professionals should be updated to include these possible very rare conditions to help them to spot potential signs and symptoms

The clotting disorders could be the result of post-covid syndrome or linked to oral contraceptives.

The decision to temporary suspend vaccinations by many European countries was exaggerated . Mahase (2021c), Mahase (2021a)..

Oxford-AstraZeneca's vaccine cost (US\$2-3) per dose .Agreement with the COVAX, facility holds good promise for equitable access for low and middle income countries, Knoll and Wonodi (2021).

\section{CoronaVac vaccine}

It is inactivated virus COVID-19 vaccine, The second COVID-19 vaccine developed in China, by Beijing-based company Sinovac . CoronaVac is safe and well tolerated in older adults. Neutralising antibody titres induced by the $3 \mu \mathrm{g}$ dose, as phase (I \& II) clinical trial in Renqiu (Hebei, China) suggested. CoronaVac was found to be (51\%) effective at preventing COVID-19 in late-stage trials. It is (100\%) effective at preventing severe disease and death. Zhiweiwu et al. (2021) It is given in two doses. Booster dose may be considered. It was approved by WHO on June, first,2021. CoronaVac is sustaining vaccination campaigns in more than forty (40) countries, such as Chile and Botswana. Globally, more than (600) million doses have been distributed Mallapaty (2021) .

Other promising Chinese vaccine is Sinopharm COVID-19 vaccine (BBIBP-CorV), was developed by Chinese company Sinopharm, its internal analysis indicated a (79\%) efficacy of the vaccine in December 2020 , in phase (III) clinical trial. It is inactivated virus COVID-19 vaccine, contains a SARS-CoV-2 strain inactivated inside Vero cells, It is established old technology based on inactivated virus. It is given in two doses. Booster dose may be considered. It could be transported and stored at normal refrigerated temperatures. Since its approval by WHO on May ,2021. Sinopharm's vaccine has been approved in many more countries.

\section{Gam-COVID-Vac vaccine (Sputnik V)}

It is recombinant (heterologous DNA) adenovirus vaccine with two different types of adenovirus vectors (rAd26 and rAd5) for the first and second dose respectively. The vaccine is composed of two different adenoviruses that produce the coronavirus spike protein, administered three weeks apart and developed by the Gamaleya National Center of Epidemiology and Microbiology in Moscow- Russia. It was registered in August 2020 by the Russian Ministry of Health as (Gam-COVID-Vac). More than sixty one (61) countries register it. 
Interpretation This interim analysis of the phase (III) trial of Gam-COVID-Vac showed (91.6\% )efficacy against COVID-19 , and (100\%) efficacy against moderate or severe COVID-19. It was well tolerated in a large cohort. Denis et al. (2021), Montalti et al. (2021)

The reported side effects repotted by health care workers in Argentine after first dose of vaccine, are pain at the injection site, redness and swelling, fever, diarrhea and muscle pain. The rate of these side effects was higher among female than male $(65.4 \%$ vs $50 \%)$ and in younger than fifyfive (55) years-old (72.8\% vs $32 \%)$. Pagotto et al. (n.d.). After receiving the Sputnik V vaccination, Telegram users complained about pain (47\%), fever (47\%), fatigue (34\%), and headache (25\%). It occurs more frequent with the first dose than from the second dose. Over all the results showed that the adverse events profile of Sputnik V was comparable with other COVID-19 vaccines and similar to other vector vaccines. They are also similar to those reported in the Argentinean post-marketing adverse events registry. Jarynowski et al. (n.d.)

In the Republic of San Marino, the ROCCA interim analysis confirmed a good tolerability profile in the over sixty (60) years age group after both doses regarding short-term solicited adverse events following immunization Montalti et al. (2021)

Challenges facing vaccine include distribution equality, skepticism and emergence of new resistant potentially virulent COVID- 19 strains .Therefore there is a potential risk of illness after successful vaccination and subsequent infection with other virulent variant strains of the virus. Continuous efforts to prevent and diagnose infection and to characterize new virus strains in vaccinated persons, should not be ignored Hacisuleyman et al. (2021).

\section{Ethical considerations}

Since minority communities and communities of colors are more affected by COVID-19, there is need for diversity in large-scale phase (III) vaccine clinical trials to include those minorities, especially in the shadow of persistent minority underrepresentation in clinical trials, even for conditions that affect them disproportionately harsh. This inclusion could build vaccine confidence in those vulnerable communities by generating data that demonstrate safety and efficacy for people like them Jaklevic (2020). There is also an urgent need for global cooperation, solidarity and appropriate regulation to ensure equitable access to a potential COVID-19 vaccine Makoni (2020)

As some countries are passive recipients of vaccines developed and tested elsewhere, misinformation and skepticism may spread there. The burden of risk is shouldered by individuals in the countries, developing and testing the new vaccines which are usually given to healthy subjects to prevent diseases. More equal representation, transparency and the involvement of local authorities and investigators in the process are therefore essential to improve trust for the recipients .

First dose priority to vaccinate as many people as possible should be adopted by decision and policy makers. It is sound logic to prioritize high risk, who have chronic 
disease and older people as first recipients of the vaccine. Also prioritization of older and younger people with the specific type of vaccine is also seemed logical. Individuals should not deny the right to chose type of vaccine if that is possible and more over obligatory vaccination may breach individuals human right. Unplanned administration of different doses exceeded licensure thresholds and mixed-dose regimen, may raised public concerns.

National Immunization Technical Advisory Groups will have to consider all factors , when taking decision on which vaccine is suitable for their setting, as regard to efficacy, safety, delivery, community acceptance, longevity of effect, whether a vaccine reduces infection and transmission as well as disease, Knoll and Wonodi (2021). If the access to a safe and effective vaccine is not wide enough, COVID-19 could turn into a poverty disease in the short term, Shay et al. (2021), with possible emergence of more virulent COVID-19 virus strains.

The second half of this year may witness approval of many more COVID-19 vaccines, as many countries take the initiative like India, Cuba and many other European counties to develop their own vaccines, beside transfer of vaccine production technology to other less develop countries in Africa. These steps should be welcomed and celebrated by global communities sincerely, rather than met by hesitancy and skepticism.

\section{RECOMMENDATION}

It is recommended to implement post-vaccination observation periods, and to continue monitoring the safety of COVID-19 vaccines to inform vaccination policy makers and to maintain public confidence .Counseling vaccine recipients, by healthcare providers, to expect transient local and systemic reactions might ease concerns and encourage completion of the second dose of vaccination. More transparency and data sharing and publishing of new vaccine development and testing results will enhance the public confidence and further intake of COVID-19 new vaccines.

\section{DISCLAIMER}

The materials here by is intended only for educational, research and reference purposes , and should not be used as medical diagnosis, neither as suitable substitute for the advice of a qualified health care professionals, and thereby no one should disregard or avoid professionals medical advice due to content published here.

\section{REFERENCES}

Alhazmi, A., Alamer, E., Daws, D., Hakami, M., Darraj, M., Abdelwahab, S., Maghfuri, A., \& Algaissi, A. (2021). Evaluation Of Side Effects Associated With Covid-19 Vaccines In Saudi Arabia. Vaccines., 9, 674. Retrieved from Https://Doi.Org/10.3390/ Vaccines 9060674 
Allergic Reactions Including Anaphylaxis After Receipt Of The First Dose Of Moderna Covid19 Vaccine - United States. (2020). Cdc Covid-19 Response Team And Food And Drug Administration, 70, 125-129.

Baden, H. M. E. L. R., Sahly, B., \& Essink. (2021). Et Al. Efficacy And Safety Of The Mrna-1273 Sars-Cov-2 Vaccine. N Engl J Med, 384(5), 403-416.

Badiani, A. A., Patel, J. A., Ziolkowski, K., \& Nielsen, F. B. H. (2020). Pfizer: The Miracle Vaccine For Covid-19?.Public Health In Practice., 1, 100061-100061. 10.1016/J.Puhip.2020 .100061

Bernal, J. L., Andrews, N., Gower, C., \& Al, E. (2021). Effectiveness Of The Pfizer-Biontech And Oxford-Astrazeneca Vaccines On Covid-19 Related Symptoms, Hospital Admissions, And Mortality In Older Adults In England: Test Negative Case-Control Study. Bmj, 373, 1088-1088.

Burd, I., Kino, T., \& Segars, J. (2021). The Israeli Study Of Pfizer Bnt162b2 Vaccine In Pregnancy: Considering Maternal And Neonatal Benefits. J Clin Invest.

Cohen, J. (2020). Vaccine Designers Take First Shots At Covid-19., 14-16.

Cucinotta, D., \& Vanelli, M. (2020). Who Declares Covid-19 A Pandemic. Acta Biomed, 91, 157160.

Denis, Y., Logunov, I. V., Dolzhikova, Dmitry, V., Shcheblyakov, E., \& Al. (2021). Safety And Efficacy Of An Rad26 And Rad5 Vector-Based Heterologous Prime-Boost Covid-19 Vaccine: An Interim Analysis Of A Randomised Controlled Phase 3 Trial In Russia. Lancet, 397, 671-81.

Edward, H., \& Livingston. (2021). Necessity Of 2 Doses Of The Pfizer Andmoderna Covid-19 Vaccines Jama March 2., 325(9).

Edward, H., Livingston, P. N., Malani, C. B., \& Creech. (2021). The Johnson \& Johnson Vaccine For Covid-19 Jama., 325, 1575-1575. Retrieved from 10.1001/Jama.2021.2927

Ej, H., Fj, A., Jm, M., \& Al, E. (2021). Impact And Effectiveness Of Mrna Bnt162b2 Vaccine Against Sars-Cov-2 Infections And Covid-19 Cases, Hospital Admissions, And Deaths Following A Nationwide Vaccination Campaign In Israel: An Observational Study Using National Surveillance Data. Lancet.

Gee, J. P., Marquez, J., Su, E., \& Al. (2020). First Month Of Covid-19 Vaccine Safety Monitoring - United States, December 14, 2020-January 13, 2021. Mmwr . February 26, 2021 ., $70(8)$.

Hacisuleyman, C., Hale, Y., Saito, E., \& Al. (2021). Vaccine Breakthrough Infections With SarsCov-2 Variants. $N$ Engl J Med, 384, 2212-2218.

Iacobucci, G. (2021). Covid-19: Two Doses Of Pfizer Vaccine Are "Highly Effective" Against Infection, Hospital Admission, And Death, Study Finds. Bmj, 373, 1164-1164.

Jaklevic, M. C. (2020). Researchers Strive To Recruit Hard-Hit Minoritiesinto Covid-19 Vaccine Trials. Jama, 324(9).

Jarynowski, A., Semenov, A., \& Kamiński, M. (n.d.). Vitaly Belik Mild Adverse Events Of Sputnik V Vaccine Extracted From Russian Language Telegram Posts Via Bert Deep Learning Model. Medrxiv.

Knoll, M. D., \& Wonodi, C. (2021). Oxford-Astrazeneca Covid-19 Vaccine Efficacy. Lancet. January 09. Lancet, 397(10269), 72-74. Retrieved from Https://Doi.Org/10.1016/S0140 -6736(20)32623-4

Leshem, E., \& Wilder-Smith, A. (2021). Covid-19 Vaccine Impact In Israel And A Way Out Of The Pandemic. Lancet.

Lipsitch, M., \& Kahn, R. (2021). Interpreting Vaccine Efficacy Trial Results For Infection And Transmission. Vaccine, 39, 4082-4088. 
Macneil, J. R., Su, J. R., Broder, K. R., \& Al, E. (n.d.). Updated Recommendations From The Advisory Committee On Immunization Practices For Use Of The Janssen (Johnson \& Johnson) Covid-19 Vaccine After Reports Of Thrombosis With Thrombocytopenia Syndrome Among Vaccine Recipients - United States, April 2021. Mmwr 2021 Apr 30., 70(17), 651-656. 10.15585/Mmwr.Mm7017e4

Mahase, E. (n.d.-a). Covid-19: Moderna Vaccine Is Nearly 95\%Effective, Trial Involving High Risk And Elderly People Shows.Bmj 2020(B);371:M4471 ., 371. 10.1136/Bmj.M4471

Mahase, E. (n.d.-b). Covid-19: Uk Approves Moderna Vaccine To Be Given As Two Doses 28 Days Apart. Bmj 2021(B), 372, 74-74.

Mahase, E. (n.d.-c). Covid-19: What Do We Know About The Late Stage Vaccine Candidates. Bmj 2020 (A), 371, 4576-4576.

Mahase, E. (2021a). Covid-19: Astrazeneca Vaccine Is Not Linked To Increased Risk Of Blood Clots, Finds European Medicine Agency. Bmj 2021(E), 372, 774-774.

Mahase, E. (2021b). Covid-19: Us Suspends Johnson And Johnson Vaccine Rollout Over Blood Clots. Bmj, 373, 970-970.

Mahase, E. (2021c). Covid-19: Who Says Rollout Of Astrazeneca Vaccine Should Continue, As Europe Divides Over Safety. Bmj, 2021, 16-16.

Mahase, E. (2021d). How The Oxford-Astrazeneca Covid-19 Vaccine Was Made. Bmj, 2021.

Makoni, M. (2020). Munyaradzi Makoni.Covid-19 Vaccine Trials In Africa. Lancet. Vol 8 November 2020 .Published Online September 4., 8. Retrieved from Https://Doi.Org/ 10.1016/S2213-2600(20)30401-X

Malayala, G. S. V., Mohan, D., \& Vasireddy. (2021). And Paavani Atluri. Purpuric Rash And Thrombocytopenia After The Mrna-1273 (Moderna) Covid-19 Vaccine. Cureus, 13(3), 14099-14099.

Mallapaty, S. (2021). Who Approval Of Chinese Coronavac Covid Vaccine Will Be Crucial To Curbing Pandemic Nature. News. 04 June.

Ma'mon, M., Hatmal, Mohammad, A. I., Al-Hatamleh, A. N., Olaimat, E., \& Al. (n.d.). Side Effects And Perceptions Following Covid-19 Vaccination In Jordan: A Randomized, CrossSectional Study Implementing Machine Learning For Predicting Severity Of Side Effects. Vaccines, 2021(6), 556-556.

Mcmahon, D. E., Amerson, E., Rosenbach, M., \& Al, E. (2021). Cutaneous Reactions Reported After Moderna And Pfizer Covid-19 Vaccination: A Registry-Based Study Of 414 Cases. J Am Acad Dermatol, 85, 46-55.

Merrynvoyseydphil, S. A., Costaclemensphd, S., Amadhiphd, E., \& Al. (2021). Safety And Efficacy Of The Chadox1 Ncov-19 Vaccine (Azd1222) Against Sars-Cov-2: An Interim Analysis Of Four Randomised Controlled Trials In Brazil, South Africa, And The Uk. Lancet., 397(10269), 99-111.

Montalti, M., Soldà, G., Valerio, Z. D., \& Al, E. (2021). Rocca Study Protocol And Interim Analysis On Safety Of Sputnik V Vaccine (Gam-Covid.Vac) In The Republic Of San Marino: An Observational Study Using Active Surveillance. Medrxiv.

Nagla, A., El-Shitany, S., Harakeh, Shaimaa, M., Badr-Eldin, E., \& Al. (2021). Minor To Moderate Side Effects Of Pfizer-Biontech Covid-19 Vaccine Among Saudi Residents: A Retrospective Cross-Sectional Study. Int J Gen Med, 14, 1389-1401.

Oliver, S. E., Gargano, J. W., \& Marin, M. (2021). The Advisory Committee On Immunization Practices' Interim Recommendation For Use Of Moderna Covid-19 Vaccine - United States, December 2020 .Mmwr . January 1., 69, 51-52.

Pagotto, V., Ferloni, A., María, M., Soriano, E., \& Al. (n.d.). Active Surveillance Of The Sputnik V Vaccine In Health Workers. Medrxiv. Preprint; Posted February 05., 2021-2021. 
Retrieved from Https://Doi.Org/10.1101/2021.02.03.21251071

Paltiel, A. D., Zheng, A., \& Schwartz, J. L. (2021). Speed Versus Efficacy: Quantifying Potential Tradeoffs In Covid-19 Vaccine Deployment. Annals Of Internal Medicine.

Pilishvili, T., Fleming-Dutra, K. E., Farrar, J. L., \& Al, E. (2021). Interim Estimates Of Vaccine Effectiveness Of Pfizer-Biontech And Moderna Covid-19 Vaccines Among Health Care Personnel - 33. Mmwr Morb Mortal Wkly Rep, 70(20), 753-758.

Sadoff, J., Gars, G. M. L., Shukarev, E., \& Al. (n.d.). Interim Results Of A Phase 1-2a Trial of Ad26.Cov2.S Covid-19 Vaccine. N Engl J Med .2021(A), 384, 1824-1835.

Sadoff, J., Gray, G., Vandebosch, A., \& Al, E. (n.d.). Safety And Efficacy Of Single-Dose Ad26.Cov2.S Vaccine Against Covid-19. N Engl J Med, 384, 2187-2201.

Safety, T. (2021). And Immunogenicity Of An Inactivated Sars-Cov-2 Vaccine (Coronavac) In Healthy Adults Aged 60 Years And Older: A Randomised, Double-Blind, PlaceboControlled, Phase 1/2 Clinical Trial. Lancet, 21(6), 803-812.

Seyed, H., Shahcheraghi, J., Ayatollahi, A. A., Aljabali, E., \& Al. (n.d.). An Overview Of Vaccine Development For Covid-19. Ther. Deliv, 2021(3), 235-244.

Shay, D. K., Gee, J., Su, J. R., \& Al. (2021). Safety Monitoring Of The Janssen (Johnson \& Johnson) Covid-19 Vaccine - United States, March-April 2021. Mmwr . May 7, 2021., 70(18), 680-684

Tanne, J. H. (2020). Covid-19: Fda Authorizes Moderna Vaccine As Us Starts Vaccinating Health Workers. Bmj, 371.

Torjesen, I. (2021). Covid-19: Astrazeneca Vaccine Is Approved In Eu With No Upper Age Limit. Bmj, 372.

Vogel, L. (2021). Feds Update Immunization Advice With Moderna Vaccine Approval . Cmaj . January 18., 193(3).

Wei, N., Fishman, M., Wattenberg, D., Gordon, M., \& Lebwohl, M. (2021). “Covid Arm”: A Reaction To The Moderna Vaccine .Jaad Case Rep., 10, 92-95.

Who. Coronavirus Disease (Covid-19) Situation Report-127. World Health Organisation (Who). (2020). Coronavirus Disease (Covid-19) Situation Report-127. World Health Organisation (Who), 1-17. Retrieved from Https://Apps.Who.Int/Iris/Handle/10665/ 332232

Zhiweiwu, Y., Profmiaoxu, E., \& Al. (2021). Safety, Tolerability, And Immunogenicity Of An Inactivated Sars-Cov-2 Vaccine (Coronavac) In Healthy Adults Aged 60 Years And Older: A Randomised, Double-Blind, Placebo-Controlled, Phase 1/2 Clinical Trial. Lancet, 21(6), 803-812. 